\title{
Numerical comparison of robustness of shaped beam delivery through multimode and multicore fibre against fibre bending
}

\author{
Veettikazhy, Madhu; Hansen, Anders Kragh; Marti, Dominik; Dholakia, Kishan
}

\section{Published in:}

Proceedings of SPIE

Link to article, DOI:

$10.1117 / 12.2542830$

Publication date:

2020

Document Version

Publisher's PDF, also known as Version of record

Link back to DTU Orbit

Citation (APA):

Veettikazhy, M., Hansen, A. K., Marti, D., \& Dholakia, K. (2020). Numerical comparison of robustness of shaped beam delivery through multimode and multicore fibre against fibre bending. In Proceedings of SPIE [112480U] SPIE - International Society for Optical Engineering. Proceedings of SPIE - The International Society for Optical Engineering https://doi.org/10.1117/12.2542830

\section{General rights}

Copyright and moral rights for the publications made accessible in the public portal are retained by the authors and/or other copyright owners and it is a condition of accessing publications that users recognise and abide by the legal requirements associated with these rights.

- Users may download and print one copy of any publication from the public portal for the purpose of private study or research.

- You may not further distribute the material or use it for any profit-making activity or commercial gain

- You may freely distribute the URL identifying the publication in the public portal 


\section{Numerical comparison of robustness of shaped beam delivery through multimode and multicore fibre against fibre bending}

Veettikazhy, Madhu, Hansen, Anders, Marti, Dominik, Dholakia, Kishan, Andersen, Peter

Madhu Veettikazhy, Anders Kragh Hansen, Dominik Marti, Kishan Dholakia, Peter Eskil Andersen, "Numerical comparison of robustness of shaped beam delivery through multimode and multicore fibre against fibre bending," Proc. SPIE 11248, Adaptive Optics and Wavefront Control for Biological Systems VI, 112480U (17 February 2020); doi: 10.1117/12.2542830 


\title{
Numerical Comparison of Robustness of Shaped Beam Delivery through Multimode and Multicore Fibre against Fibre Bending
}

\author{
Madhu Veettikazhy ${ }^{\mathrm{a},},{ }^{,}$, Anders Kragh Hansen ${ }^{\mathrm{b}}$, Dominik Martia ${ }^{\mathrm{a}}$, Kishan Dholakia ${ }^{\mathrm{c}, \mathrm{d}}$, and Peter \\ Eskil Andersen ${ }^{\mathrm{a}}$ \\ ${ }^{a}$ DTU Health Tech, Technical University of Denmark, Frederiksborgvej 399, 4000 Roskilde, \\ Denmark \\ bDTU Fotonik, Technical University of Denmark, Frederiksborgvej 399, 4000 Roskilde, \\ Denmark

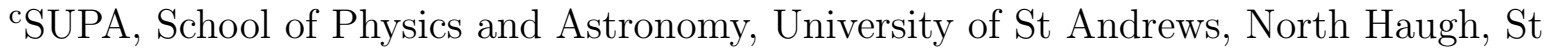 \\ Andrews, KY16 9SS, UK \\ ${ }^{\mathrm{d}}$ Department of Physics, College of Science, Yonsei University, Seoul 03722, South Korea
}

\begin{abstract}
Miniaturisation of endoscopes can be achieved using lensless endoscope probes, which enhances in vivo deeptissue imaging technology. The necessity of a detailed understanding of light propagation through optical fibres is paramount, since beam focusing and scanning at tissue require beam shaping at the proximal end of the fibre. For stable light delivery and collection, the sensitivity of various fibre profiles against fibre deformations needs to be reviewed. We present a numerical simulation tool investigating optical field propagation through multimode and multicore optical fibres, emphasizing fibre-bending deformations. The simulation tool enables user to choose optimum fibre with best possible realistic parameters for any application.
\end{abstract}

Keywords: Optical fibre, fibre simulations, light-sheet microscopy, wavefront control, fibre bending, beam propagation method, multicore fibre

\section{INTRODUCTION}

Lensless endoscopes provide a means for delivery and collection of light to and from tissue without any need for additional optical elements at the fibre distal end.$^{1}$ These facilitate to reduce the overall footprint of the device to the order of an optical fibre, which potentially leads to easier non-invasive deep-tissue imaging. Essentially, an extensive analysis of the laser beam propagation through the optical fibre is required in this case, since any focusing or scanning at the sample site requires the beam to be shaped at the fibre's proximal end. For an accurate imaging system, fiber bending must not affect the beam delivery and subsequent collection, or at least do so in a way that is easy to detect and for which compensation can be applied. Given this background, one could experimentally acquire the Transformation Matrix (TM) of the straight fibre at hand that could help determine optical propagation even in non-ideal fibre structures,${ }^{2,3}$ but this method is helpful only if one has experimental TM results corresponding to a specific existing fibre and the method does not help in a theoretical investigation in which optimal fibre parameters are sought. To this end, we present a numerical tool to simulate various types of optical fibre under the realistic use case of bending deformations, which in turn allows the user to choose the best optical fibre with suitable parameters for the specific application before starting the experiment.

\footnotetext{
*Corresponding author: madve@dtu.dk
} 


\section{THEORY}

The numerical simulations of optical field propagation through varied profiles of optical fibres were performed using the finite difference beam propagation method (FDBPM).$^{4,5}$ The paraxial Helmholtz equation can be represented as:

$$
2 j k_{0} n_{0} \frac{\partial E}{\partial z}=\frac{\partial^{2} E}{\partial x^{2}}+\frac{\partial^{2} E}{\partial y^{2}}+k_{0}^{2}\left[n^{2}(x, y, z)-n_{0}^{2}\right] E
$$

where $E=E(x, y, z)$ is the electric field inside the optical fibre with refractive index profile represented as $n(x, y, z)$. Using the finite difference approximation, Eq. (1) can be written as:

$$
2 j k_{0} n_{0} \frac{\partial E_{p, q}}{\partial z}=\frac{E_{p-1, q}-2 E_{p, q}+E_{p+1, q}}{\Delta x^{2}}+\frac{E_{p, q-1}-2 E_{p, q}+E_{p, q+1}}{\Delta y^{2}}+k_{0}^{2}\left[n_{p, q}^{2}(z)-n_{0}^{2}\right] E_{p, q},
$$

where $E_{p, q}$ is the electric field at $(p \Delta x, q \Delta y, z)$ with $p=0,1,2, \cdots, N_{x}-1, q=0,1,2, \cdots, N_{y}-1$, and $N_{x}, N_{y}$ being the number of pixels in $x$ and $y$ directions. Integrating Eq. (2) in the interval $[z, z+\Delta z]$ and application of trapezoidal rule of integration lead to the Crank-Nicolson finite difference representation of the relation between electric fields at transverse planes $z$ and $z+\Delta z$ as:

$$
\begin{aligned}
& -a_{x} E_{p-1, q}(z+\Delta z)-a_{x} E_{p+1, q}(z+\Delta z)-a_{y} E_{p, q-1}(z+\Delta z)-a_{y} E_{p, q+1}(z+\Delta z)+b E_{p, q}(z+\Delta z) \\
& \quad=a_{x} E_{p-1, q}(z)+a_{x} E_{p+1, q}(z)+a_{y} E_{p, q-1}(z)+a_{y} E_{p, q+1}(z)+c E_{p, q}(z)
\end{aligned}
$$

where

$a_{x}=\frac{\Delta z}{2 \Delta x^{2}}, a_{y}=\frac{\Delta z}{2 \Delta y^{2}}, b=2 j k_{0} n_{0}+\frac{2 \Delta z}{\Delta x^{2}}-\frac{k_{0}^{2} \Delta z}{2}\left(n_{p, q}^{2}(z+\Delta z)-n_{0}^{2}\right)$, and $c=2 j k_{0} n_{0}-\frac{2 \Delta z}{\Delta x^{2}}+\frac{k_{0}^{2} \Delta z}{2}\left(n_{p, q}^{2}(z)-n_{0}^{2}\right)$. Representing $E$ as a column vector, this system of linear equations can be represented in matrix format as:

$$
\mathbf{A} \mathbf{E}^{z+\Delta z}=\mathbf{B} \mathbf{E}^{z}
$$

where

$$
\mathbf{A}=\left[\begin{array}{cccccccccc}
b & -a_{x} & 0 & \cdots & 0 & -a_{y} & 0 & & \\
-a_{x} & b & -a_{x} & 0 & & 0 & -a_{y} & 0 & & \\
0 & -a_{x} & b & -a_{x} & 0 & & 0 & -a_{y} & 0 & \\
\vdots & 0 & -a_{x} & b & -a_{x} & 0 & & 0 & \ddots & \\
0 & & 0 & \ddots & \ddots & \ddots & 0 & & \\
-a_{y} & 0 & & 0 & & & & & & \\
0 & -a_{y} & 0 & & & & & & \ddots & \\
& 0 & -a_{y} & 0 & & & & & \ddots & -a_{x} \\
& & 0 & \ddots & & & & \ddots & \ddots & \\
& & & & & & & & -a_{x} & b
\end{array}\right], \quad \text { and }
$$

$$
\mathbf{B}=\left[\begin{array}{cccccccccc}
c & a_{x} & 0 & \cdots & 0 & a_{y} & 0 & & \\
a_{x} & c & a_{x} & 0 & & 0 & a_{y} & 0 & & \\
0 & a_{x} & c & a_{x} & 0 & & 0 & a_{y} & 0 & \\
\vdots & 0 & a_{x} & c & a_{x} & 0 & & 0 & \ddots & \\
0 & & 0 & \ddots & \ddots & \ddots & 0 & & & \\
a_{y} & 0 & & 0 & & & & & & \\
0 & a_{y} & 0 & & & & & & & \\
& 0 & a_{y} & 0 & & & & & \ddots & \\
& & 0 & \ddots & & & & \ddots & \ddots & a_{x} \\
& & & & & & & a_{x} & c
\end{array}\right]
$$



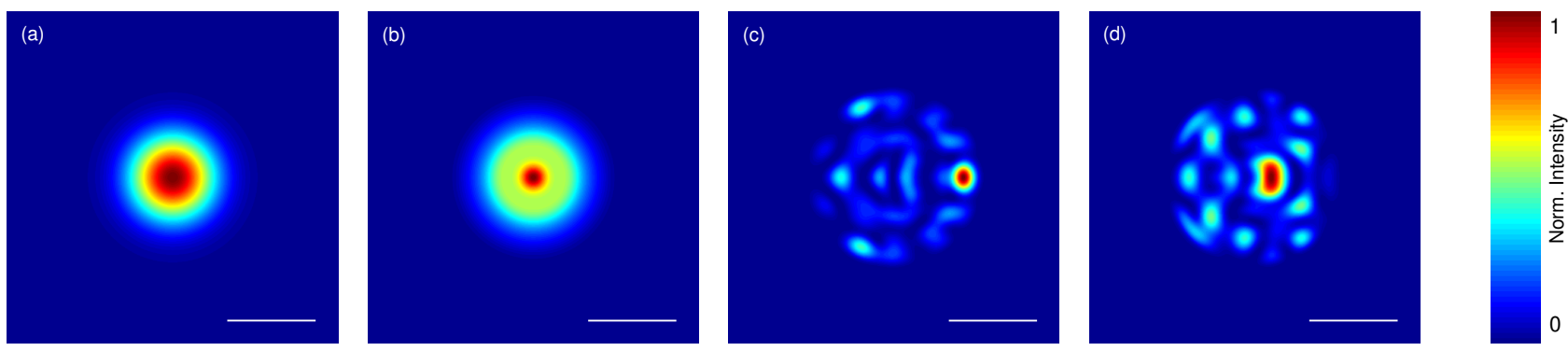

Figure 1. (a) and (b) represent the intensity profiles of the optical field at the proximal and distal ends of the straight multimode fibre. (c) and (d) represent the intensity profiles of the optical field at the distal end of multimode fibre which is bent $10^{\circ}$ and $20^{\circ}$ respectively with bending radius of $1 \mathrm{~cm}$. Scalebars: (a)-(d) $20 \mu \mathrm{m}$.
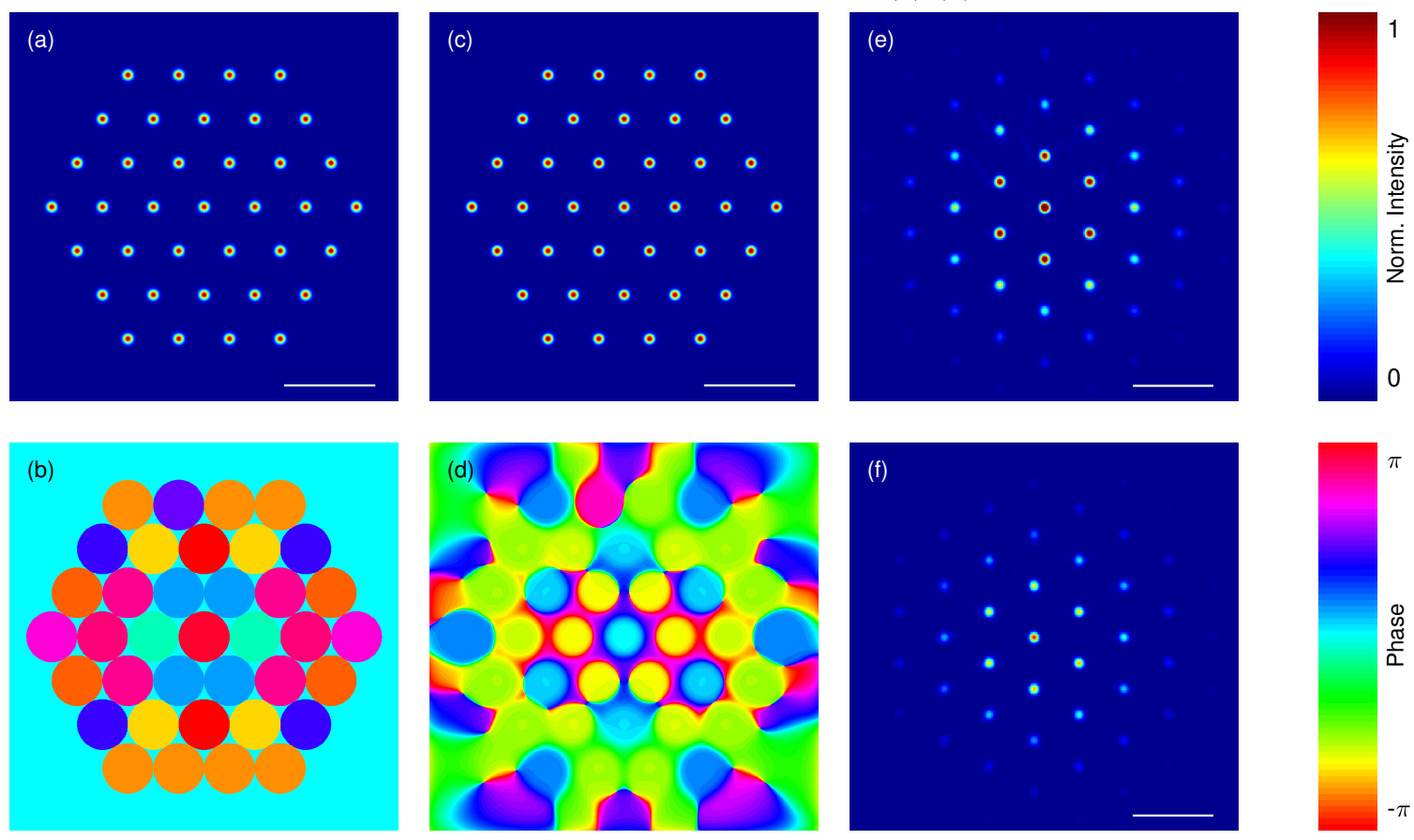

Figure 2. (a) and (b) represent the intensity and phase of the optical field at the straight multicore fibre proximal end. (c) and (d) represent the intensity and phase of the optical field at the straight multicore fibre distal end. (e) represents the intensity of the optical field at a transverse plane $500 \mu \mathrm{m}$ in front of the straight multicore fibre distal end. (f) represents the intensity of the optical field at a transverse plane $500 \mu \mathrm{m}$ in front of the distal end of multicore, which is bent $20^{\circ}$ with bending radius of $1 \mathrm{~cm}$. Scalebars: (a)-(d) $25 \mu \mathrm{m},(\mathrm{e}, \mathrm{f}) 50 \mu \mathrm{m}$.

are sparse matrices of order $N \times N$, where $N=N_{x} * N_{y}$. As in matrices A and B in Eq. (5), the displaced diagonals start after $N_{x}$ rows or columns, which correspond to the partial differentiation in $y$ direction. Also, in the two diagonal entries close to the principal diagonal, every $N_{x}^{\text {th }}$ entry will be 0 , accounting for the Dirichlet's boundary condition. Finally, the matrix system can be solved to find the electric field at the transverse plane $z+\Delta z$ as:

$$
\mathbf{E}^{z+\Delta z}=\mathbf{A}^{-1} \mathbf{B} \mathbf{E}^{z}
$$

\section{NUMERICAL SIMULATION RESULTS}

Multimode and multicore fibres were simulated using the described FDBPM method by choosing the refractive index $n(x, y, z)$ profile accordingly. The intensity of the electric field before and after propagation through a 
straight $15 \mathrm{~cm}$ long multimode fibre, which supports 229 modes, is represented in Fig. 1(a,b). Fig. 1(c,d) show the intensity profiles of the field at the distal end of the multimode fibre with bending angles $10^{\circ}$ and $20^{\circ}$ and bending radius $1 \mathrm{~cm}$.

The intensity and phase profiles of the electric field before and after propagation through a multicore fibre of 37 single mode cores are represented in Fig. 2(a)-(d). The field distribution was simulated to propagate a distance of $15 \mathrm{~cm}$ through the multicore fibre. The phase profile in Fig. 2(b) of the output electric field is solely responsible for the focusing effect after the distal end, as seen in Fig. 2(e,f). We could, for instance, model an optical fibre to be used for light-sheet microscopy, where generating a focus at the fibre distal end is paramount. Structured illumination at the distal end, such as a line focus, can be achieved by shaping the beam through individual cores of the modeled multicore fibre.

\section{DISCUSSIONS}

The speckle-like patterns shown in Fig. 1 (c,d) are consistent with the beam patterns often experimentally achieved when bending a multimode fibre. This critical behaviour poses a significant challenge when beam shaping through a multimode fibre.

As shown in Fig. 2(e), the optical field propagated through the fibre could be focused at a short distance in front of the fibre distal end without using any focusing optics. One could also observe that once the multicore fibre is bent through an angle of $20^{\circ}$ with bending radius $1 \mathrm{~cm}$, the intensity pattern at the focal plane will be slightly shifted from that of a non-deformed fibre, as shown in Fig. 2(f). At the conference, we will present results comparing the robustness against fibre bending of multimode and that of multicore fibres and show how this simulation tool enables the user to choose an optimum optical fibre with the best possible realistic parameters for any particular application.

\section{REFERENCES}

[1] Andresen, E. R., Sivankutty, S., Tsvirkun, V., Bouwmans, G., and Rigneault, H., "Ultrathin endoscopes based on multicore fibers and adaptive optics: a status review and perspectives," Journal of Biomedical Optics 21(12), 1 - 12 (2016).

[2] Plöschner, M., Kollárová, V., Tsvirkun, V., Dostál, Z., Nylk, J., Barton-Owen, T., Ferrier, D. E. K., Chmelík, R., Dholakia, K., and Čižmár, T., "Multimode fibre: Light-sheet microscopy at the tip of a needle," Scientific Reports 5, 18050 (2015).

[3] Plöschner, M., Tyc, T., and Čižmár, T., "Seeing through chaos in multimode fibres," Nature Photonics 9, $529-535(2015)$.

[4] Chung, Y. and Dagli, N., "An assessment of finite difference beam propagation method," IEEE Journal of Quantum Electronics 26, 1335-1339 (Aug 1990).

[5] Yevick, D. and Hermansson, B., "Efficient beam propagation techniques," IEEE Journal of Quantum Electronics 26, 109-112 (Jan 1990). 